\title{
Patient bedside observations: what could be simpler?
}

\section{Michael Buist, Stella Stevens}

The Centre for Health Services Research, School of Medicine, University of Tasmania, Hobart, Tasmania, Australia

\section{Correspondence to} Prof Michael Buist, The Centre for Health Services Research, School of Medicine, University of Tasmania, Private Bag 3513, Burnie, TAS 7320, Australia; Michael.Buist@utas.edu.au

Received 13 May 2013 Accepted 13 May 2013 Published Online First 31 May 2013

\section{SLinked}

- http://dx.doi.org/10.1136/ bmjqs-2013-001954

To cite: Buist M, Stevens S. BMJ Qual Saf 2013;22:699701.

\section{INTRODUCTION}

Most readers will understandably pass by the piece by Hands et al, "Patterns in the recording of vital signs and early warning scores [EWSs]: the degree of compliance with a clinical escalation protocol." The keywords 'patterns', 'recording', 'vital signs', 'early warning scores', 'protocol' and 'compliance' will attract only the most interested in this area. A complicated-looking methodology and the use of technical jargon and acronyms may even prevent skimming by casually interested readers. Even experts in this topic may pass over the paper, perhaps just giving it a quick glance and nodding in agreement that 'we all know'patient observations are either not taken or are taken incorrectly and that when they are taken and recorded correctly few clinicians take any interest in them. ${ }^{2}$

\section{STUDY FINDINGS}

That said, the prosaic sounding title and technical jargon belie the fundamental contribution this research represents with respect to efforts to improve the safety and quality of hospital care. To justify that claim we need to explain in plain English what the authors have done. Hands et $a l^{1}$ assembled a massive cohort including the vital signs of all adult patients over a 1-year period in a large National Health Service (NHS) District General Hospital in the UK using bedside observation data captured on an electronic system. This allowed for the analysis of nearly 1 million patient observation sets. They then asked two fundamental questions: first, what is the pattern of the observation capture (eg, how frequently do patients have vital signs checked, what proportions are abnormal and do abnormal recordings occur more frequently at certain times of day)? And second, but more importantly, to what extent do staff adhere to hospital policy such that abnormal findings trigger further, more frequent observations?
By way of background, it is important to understand that most NHS hospitals have some sort of EWS that is a numeric aggregate of the weighted values that apply to abnormalities of the basic bedside observations. The more abnormal the observations, the higher the EWS, and in most protocols, higher scores trigger more frequent observations. This group from Portsmouth call their EWS ViEWS as it was derived from the proprietary system that they helped develop (VitalPAC). ${ }^{3}$ As with most hospital EWS procedures, ViEWS includes action or treatment algorithms such that abnormal observations should be rechecked at more frequent intervals.

So, the key findings with respect to the pattern of observation capture are as follows: there was good adherence to observation capture during the day but compliance at night was poor. With respect to the issue of compliance with the rechecking of abnormal observations: at night nearly half of patients who had a ViEWS of 7-8, which should trigger the rechecking of the observation set within $1 \mathrm{~h}$, did not have any observations performed in the next $6 \mathrm{~h}$. For those patients with a ViEWS of 9 or more, the majority of whom must have been critically unstable, nearly a third at night did not have their observations rechecked in the next $6 h^{1}{ }^{1}$

When considering these findings, one needs to understand that the data were collected in a hospital where an electronic data collection system was used and that the research team at Portsmouth have a long and distinguished track record in all areas relating to the clinically deteriorating patient. ${ }^{4}$ We could speculate that this failure to recheck patient abnormal observations at night is at best similar throughout NHS and quite probably worse.

\section{STUDY SIGNIFICANCE}

To understand the real significance of this work, we need to think about the 
fundamentals of patient bedside observations. Florence Nightingale first highlighted the importance of basic patient observations in her book, 'Notes on Nursing: What it is and What it is not. ${ }^{5}$ The simplest concept is that the absence of patient observations indicates that the patient is deceased. Conversely, the presence of observations indicates life. However, patient observations are not an all or nothing phenomenon; there is widely accepted agreement on what is normal and what is abnormal. Put simply, an abnormal observation indicates that there is something wrong physiologically with the patient. Thus, the abnormal observation alerts the treating clinical team to an issue that either needs management or more frequent observations to determine whether the observation normalises or deteriorates. In an ideal world, all patient observations occur at a frequency appropriate to the patient's condition, the observations are collected accurately and documented legibly, abnormal observations are notified to appropriate clinical staff who attend the patient in a timely fashion, manage the condition, the observations normalise and the patient gets better. Alternatively, such deterioration in patient observations could indicate pain or impending death so that appropriate palliative and comfort care can be put into place for the dying patient. What could be more simple? If only it were that easy.

Just last week in an Australian hospital a 90-year-old woman was admitted from a nursing home (where she had a 'not for resuscitation order'), underwent a fixation of a fractured hip, had infrequent observations taken overnight, was found pulseless in the morning, and then had $6 \mathrm{~min}$ of cardiopulmonary resuscitation before a relative could be contacted and resuscitation ceased. Sadly, this scenario is not uncommon: infrequent monitoring, incomplete observations, no observation plan and no intervention for abnormal observations. ${ }^{2} 7$ Hands et $a l^{1}$ provide evidence of the time periods when this patient safety issue is most likely to occur. As such, their study points the way to tackling this issue most effectively.

This is important. If one accepts that the final common pathway for clinical deterioration will in some way manifest as an abnormal observation, then the ultimate defence for harm from patient adverse events needs to be the monitoring and acting upon such abnormalities. So, the critical question is why does this not occur reliably in our healthcare systems? Fundamentally, we as healthcare professionals may have lost sight or perhaps never really understood our core business. Our view of core business is looking after patients and keeping them safe throughout their hospital stay. Regardless of all the complex and fantastic clinical innovations that we have available, our core business will always come down to that person in a hospital bed, the most junior attending doctor and the bedside nurse.
Just last night a patient was referred to one of us (MB) with acute renal failure secondary to relative hypotension (normal treated blood pressure of 130 to $140 \mathrm{~mm} \mathrm{Hg}$ systolic, and in hospital readings of between 90 and $100 \mathrm{~mm} \mathrm{Hg}$ systolic) secondary to sepsis from an infected breast tissue expander postmastectomy for breast cancer. Fortunately, the overnight intern called the Medical Emergency Team ${ }^{6}$ and I was notified. The patient was transferred to the intensive care unit, resuscitated, lines inserted and vasoconstrictors started to normalise the patient's blood pressure, with what is now improving renal function. All the clinicians involved in the patient's prior care could give me very detailed information about the management of the breast cancer, including exhaustive detail on the chemotherapy, radiotherapy and surgical management, even down to tumour receptor status. Yet, for days, they had been oblivious to the fact that the patient had abnormal observations and was slowly but surely slipping into acute renal failure secondary to sepsis. What good would all that sophisticated, multidisciplinary, cancer management have been if the patient had died from this easily detected and reversible condition?

If our core business consists of watching over that patient in the bed attended by the most junior of medical and nursing staff, then it follows that the most important tool in the delivery of our core business is patient observations. However, what we have in the real world is a failure to understand that core business. As such what we get with observation capture, understanding and action is the relegation of this duty to the most junior of nursing staff or worse, nurse assistants, and the notification of abnormalities via a crude system of 'clinical futile cycles' upwards through a traditional hierarchal referral model of care, such that important information about the patient is invariably lost in the noise and business of a day-to-day hospital. ${ }^{8}$ The people-outreach teams, acute care physicians, hospitalists and intensivistswho understand the significance of these observation abnormalities are often the last to hear about them.

Thus, we come to the real significance of this work by Hands et al. ${ }^{1}$ The electronic capture of the patient observations allows for real-time analysis of patient risk for an overall hospital system down to the individual patient ${ }^{9}$ and at the same time should enable compliance with organisational policy and procedure for abnormal observations by linking of the electronic abnormal alert to the most appropriate clinical response. ${ }^{10}$ Also, the use of an electronic data capture system that the authors have developed allows for the collection of a massive number of patient data points all in real time. To do this type of study manually from paper observation charts and retrospectively entering the data into some sort of research database would be a tiresome, thankless and frequently inaccurate task. ${ }^{11}$ Electronic monitoring is the only effective 
alternative, but the Hands et al study shows us that even that is not enough. However, by providing evidence of the time periods when this patient safety issue is most likely to occur, it enables us to target this high-risk time period.

We have to find out what happens at night when nurses record observations that indicate that a patient is moving towards the point at which an intervention is necessary. Whether the hospital monitoring system uses numerical ranges or coloured bands that are supposed to alert less-experienced staff that help is needed, we can conclude from the study by Hands et $a l^{1}$ that it doesn't achieve this objective at night. Do night-time staff make the call or try to ameliorate the situation themselves based on the tacit understanding that few medical staff are available to do 'anything' anyway? Or are they still afraid of 'disturbing' those who are available? Hands et al tell us that observations are not made at night when they should be and hence action not taken, but we need more research to find out why not. There may be an unstated understanding by night staff that, for example, when blood pressure falls into the danger zone more fluids are administered until the day shift or a doctor arrives. Or it may be, as Hands et al suggest, a case of competing clinical priorities, although this is hard to accept when we know that no patients are being discharged, few are having diagnostic tests or allied health professional treatment or even falling during this period of time.

Qualitative research, such as interviews with staff and perhaps (disguised) ethnographic observation, may shed light on what transpires at night and characterises how nurses make decisions about what to do in the face of abnormal bedside observations. For now, the study by Hands et $a l^{1}$ convincingly demonstrates that even in a hospital with a well-functioning electronic system for recording bedside observations and a mature, internally developed EWS for acting on these data, non-adherence to basic protocols for concern/observation of patients with worrying vital signs remains common.

In a statement that foreshadowed the system perspective on patient safety by over a century, Florence Nightingale also had this to say in her Notes on Nursing: 'Let whoever is in charge keep this simple question in her head (not, how can I always do this right thing myself, but) how can I provide for this right thing to be always done? ${ }^{5}$ Until we figure out how to provide for the right thing consistently occurring when it comes to our core business of watching over patients and taking appropriate action in the face of worrisome signals, it is difficult to imagine making good on Nightingale's more famous first principle for hospitals, namely that they should do the sick no harm.

Contributors M B and SS contributed equally to this paper.

Competing interests Professor Buist is the founder, patent holder, former director and current shareholder in Patientrack, a company that produces devices related to bedside assessment protocols, such as early warning scores, that is in competition with VitalPAC, the company with which some of the authors of the companion research article are affiliated.

Provenance and peer review Commissioned; internally peer reviewed.

Editor's note The editors noted the potential for conflict-an editorialist affiliated with a company in competition with the company with which some of the authors of the companion research article have affiliations. Professor Buist fully disclosed this potential conflict. Given the favourable nature of the comments in the editorial and the absence of statements about any commercial products potentially related to the subject matter of either paper, the editors did not regard the potential conflict as problematic.

\section{REFERENCES}

1 Hands C, Reid E, Meredith P, et al. Patterns in the recording of vital signs and early warning scores: the degree of compliance with a clinical escalation protocol. BMJ Qual Saf 2013;22:719-726.

2 McGain F, Cretikos MA, Jones D, et al. Documentation of clinical review and vital signs after major surgery. MJA 2008;189:380-3.

3 Smith GB, Prytherch DR, Schmidt P, et al. Hospital wide physiological surveillance-a new approach to the early identification and management of the sick patient. Resuscitation 2006;71:19-28.

4 Smith GB. In-hospital cardiac arrest: is it time for an in-hospital "chain of prevention?" Resuscitation 2010;81:1209-11.

5 Nightingale F. Notes on hospitals. 3rd edn. London: Longman green Robert and Green, 1863.

6 Buist M, Moore G, Bernard S, et al. Effects of the Medical Emergency Team in the reduction of the incidence and the mortality from unexpected in-hospital cardiac arrest calls: a preliminary study. BMJ 2002;324:387-90.

7 National Confidential Enquiry into Patient Outcomes and Death. Time to Intervene? A review of patients who underwent cardio pulmonary resuscitation as a result of an in-hospital cardiorespiratory arrest. London, 2012. http://www.ncepod.org. uk/2012cap.htm

8 Buist M, Harrison J, Abaloz E, et al. Six year audit of cardiac arrests and medical emergency team calls in an outer metropolitan teaching hospital. BMJ 2007;335:1210-12.

9 Prytherch D, Smith GB, Schmidt PE, et al. ViEWS-towards a national Early Warning Score for detecting adult inpatient deterioration. Resuscitation 2010;81:932-7.

10 Jones S, Mullally M, Ingleby S, et al. Bedside electronic capture of clinical observations and automated clinical alerts to improve compliance with a NHS Trust Early Warning Score (EWS) protocol. Crit Care Resusc 2011;13:83-8.

11 Buist M, Nguyen T, Moore G, et al. Association between clinically abnormal bedside observations and subsequent in-hospital mortality: a prospective study. Resuscitation 2004;62:137-41. 